\title{
Influence of Epidural Ropivacaine with or without Dexmedetomidine on Postoperative Analgesia and Patient Satisfaction after Thoraco-Lumbar Spine Instrumentation: A Randomized, Comparative, and Double-Blind Study
}

\author{
Faisal Qureshi ${ }^{1}$, Shyam Charan Meena ${ }^{1}$, Vishal Kumar ${ }^{2}$, Kajal Jain ${ }^{1}$, Rajeev Chauhan ${ }^{1}$, Ankur Luthra ${ }^{1}$ \\ ${ }^{I}$ Department of Anaesthesia and Intensive Care, Postgraduate Institute of Medical Education and Research, Chandigarh, India \\ ${ }^{2}$ Department of Orthopaedic surgery, Postgraduate Institute of Medical Education and Research, Chandigarh, India
}

Study Design: This was a prospective, randomized, and double-blind study.

Purpose: Thoraco-lumbar spine surgery is associated with severe postoperative pain and can cause chronic pain. We aimed to compare the impact of epidural ropivacaine with and without dexmedetomidine on postoperative analgesia after thoracolumbar spine instrumentation wherein an epidural catheter was placed by the surgeon intraoperatively.

Overview of Literature: Very few studies have reported the use of epidural dexmedetomidine in spine surgeries. When used via the epidural route, dexmedetomidine is safe and efficacious and is associated with reduced rescue analgesia consumption, increased duration of analgesia, reduced pain scores, but not with major hemodynamic adverse effects.

Methods: Total 60 American Society of Anesthesiologists I-III adult patients aged 18-65 years who were scheduled to undergo thoraco-lumbar spine instrumentation were randomly allocated into group RD (epidural ropivacaine+dexmedetomidine) or group R (epidural ropivacaine plus saline). We aimed to compare the total rescue analgesic consumption on postoperative day 0,1 , and 2. Moreover, we studied the time to first rescue analgesia with visual analogue scale score $<4$ and the overall patient satisfaction scores.

Results: There was no difference between the demographic characteristics of the two groups. The mean value of total rescue analgesia consumption was $162.5 \pm 68.4 \mathrm{mg}$ in the RD group and $247.5 \pm 48.8 \mathrm{mg}$ in the $\mathrm{R}$ group. The mean time to first rescue analgesia was $594.6 \pm 83.0$ minutes in the $\mathrm{RD}$ group and $103.6 \pm 53.2$ minutes in the $R$ group. The mean patient satisfaction score was $4.2 \pm 0.7$ in the $\mathrm{RD}$ group and $3.2 \pm 0.6$ in the $\mathrm{R}$ group. No patient had any respiratory depression or prolonged motor blockade during the postoperative period.

Conclusions: This study demonstrated the superior efficacy, in terms of postoperative analgesia and patient satisfaction scores, of epidural ropivacaine plus dexmedetomidine over that of ropivacaine alone in patients undergoing surgery for thoraco-lumbar spine.

Keywords: Thoracolumbar spine surgery; Epidural analgesia; Dexmedetomidine; Pedicle screw fixation; Postoperative pain

Received Feb 17, 2020; Revised Apr 1, 2020; Accepted Apr 20, 2020

Corresponding author: Shyam Charan Meena

Department of Anaesthesia and Intensive Care, Postgraduate Institute of Medical Education and Research, Chandigarh, Sector 12, 160012, Chandigarh, India

Tel: +91-7891669817, E-mail: drshyam.pgi@gmail.com 


\section{Introduction}

Increased automation and mechanized work along with increase in sedentary life style has led to an increase in spine pathology. However, with technological advancements, instrumentation, and the availability of better facilities in modular operation theatres, more spine surgeries have been performed in the previous few decades [1]. Patients who undergo spine surgery experience moderate to severe postoperative pain that lasts for an average of 3 days [2]. In such cases, postoperative pain may lead to complications, such as increased morbidity and mortality, and may delay postoperative recovery, resulting in increased cost of health [3]. Moreover, many spine surgery patients experience pain preoperatively; this may complicate their postoperative course if not managed in a procedural manner. Moreover, considering the fact that preoperative and persistent postoperative pain are risk factors for the development of chronic back pain syndrome, there is a significant need for management of postoperative analgesia especially in this group of patients [4].

Currently, there is no set "gold standard" for postoperative analgesia in patients undergoing thoracolumbar spine instrumentation, and rescue therapy mainly consists of conventional intermittent injection of intravenous opioids. Aggressive use of opioids may lead to over sedation, respiratory depression, urinary retention, constipation, and opioid-induced hyperalgesia [5].

Epidural drug route provides excellent analgesia and is associated with decreased occurrence of respiratory depression and sedation. Ropivacaine is a good choice for epidural analgesia in the postoperative period because it is associated with lower Visual Analog Scale (VAS) scores and postoperative rescue analgesia consumption [3]. The use of ropivacaine has an advantage in that it causes decreased incidence of motor blockade (differential blockade) and cardiotoxicity compared to bupivacaine at equivalent doses [6]. Alpha agonists, such as clonidine and dexmedetomidine, are very well suited for use in the perioperative period because of their sedative and analgesic properties. Moreover, their central sympatholytic action is desirable in the perioperative period. Dexmedetomidine is 8 times more specific than clonidine for alpha-2 agonistic activity. This makes it a choice with a preferable adverse-effect profile because of less hemodynamic upsets due to selective alpha-2 agonist activity [7].

Few previous studies have reported on the use of epi- dural ropivacaine with dexmedetomidine as a continuous infusion in thoracolumbar spine surgeries. Therefore, we designed this prospective, randomized, double-blind study to compare the impact of epidural ropivacaine with and without dexmedetomidine on postoperative analgesia and patient satisfaction scores after thoracolumbar spine instrumentation, wherein an epidural catheter was placed by the surgeon intraoperatively.

\section{Materials and Methods}

After obtaining approval of the Institutional Ethical Committee of Postgraduate Institute of Medical Education and Research, Chandigarh, India (IRB approval no., INT/ IEC/2018/001887), it was proposed that 62 patients who fulfill the following inclusion criteria be enrolled: age 1865 years; and American Society of Anesthesiologists (ASA) I-III status, scheduled for laminectomy, posterior decompression, and/or pedicle screw fixation at one or two levels of thoraco-lumbar spine instrumentation. We have taken the written informed consent for using of patients study data and the images for research and publishing purpose.

\section{Group allocation and blinding}

The patients were randomized to either the $\mathrm{R}$ group (epidural ropivacaine only) or the RD group (epidural ropivacaine with dexmedetomidine) before the surgery. Computer-generated random numbers contained in opaque, sealed envelopes were used for randomization (Fig. 1). It was given to the anesthesiologist who then prepared the study solution and was not involved in the study. The patients and the primary investigator involved in the study who followed up the cases in the recovery period for postoperative pain and other parameters were blinded to group allocation and drug preparation. The surgeon who had inserted the epidural catheters was also unaware of the study drug preparation. Hence, double blinding was ensured in this study.

\section{Anesthesia technique}

Injection morphine $(0.1 \mathrm{mg} / \mathrm{kg})$ was used for analgesia; injection propofol $(1-2.5 \mathrm{mg} / \mathrm{kg})$ was used for loss of consciousness, and vecuronium injection $(0.1 \mathrm{mg} / \mathrm{kg})$ was given for muscle relaxation. The patients were then turned in the prone position. After prone positioning, eyes and 


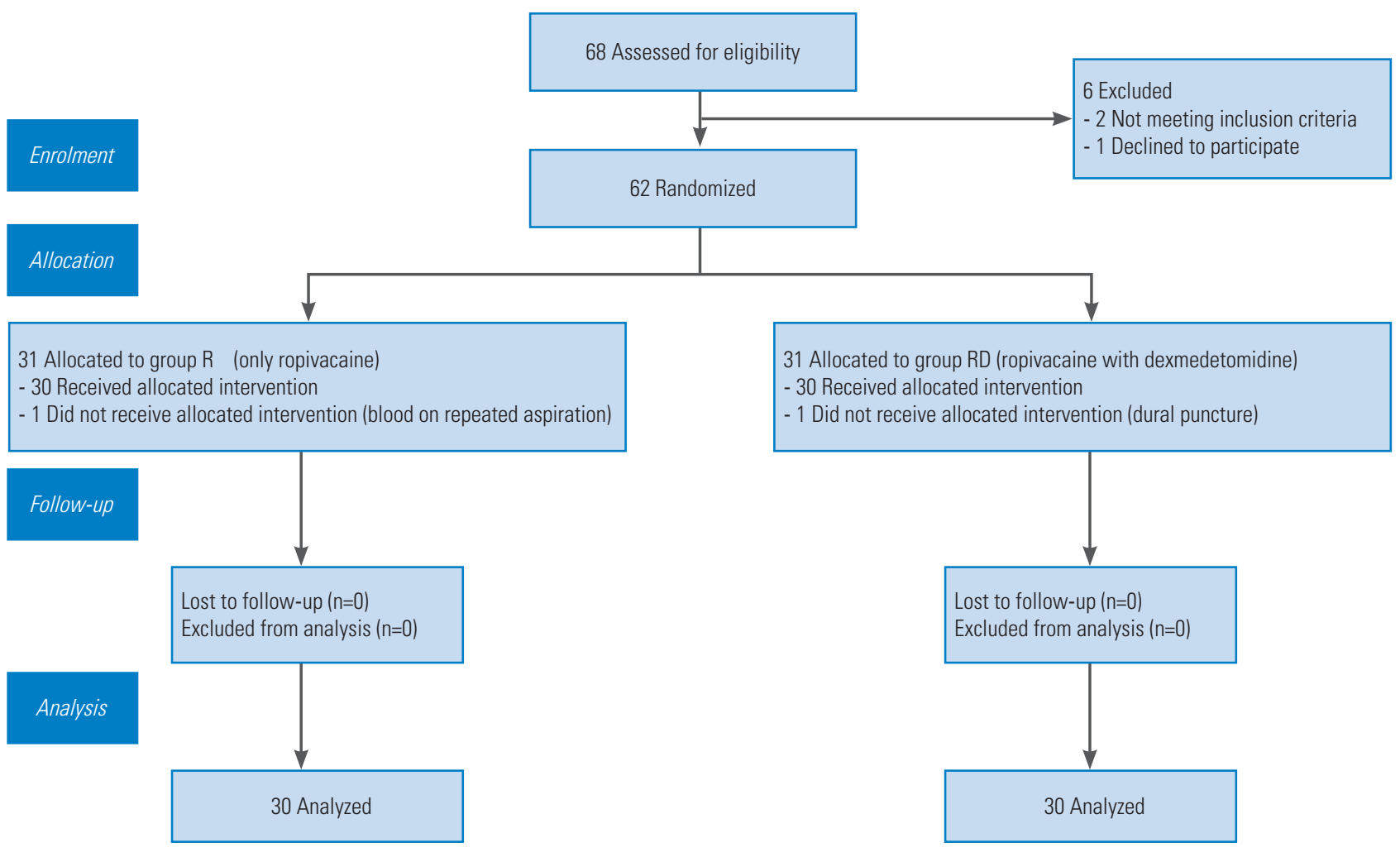

Fig. 1. Consort chart.

genitals were checked for any direct compression, and air entry was reconfirmed in the bilateral lung field.

All the surgeries were performed by the same surgeon who had previous experience of $>5$ years in spine surgery. Surgical approach was standard posterior, midline with sub periosteal elevation, and retraction of the para spinal muscles. The surgical level was determined clinically and ascertained under image intensifier. Posterior decompression with laminectomy of the concerned level was performed, and a rod of applicable length was inserted along the spinous processes. Finally, pedicle screws were inserted as per the anatomical landmarks under the guidance of image intensifier. After completion of the surgical procedure and before closure of the surgical wound, under all aseptic precautions, a Portex multi-hole epidural catheter was placed under direct vision in the epidural space through a separate skin puncture above the incision (about $3 \mathrm{~cm}$ above the main surgical incision in the midline of the spine) with $18 \mathrm{G}$ Tuohy's needle. The catheter was tunneled subcutaneously and positioned up to $13 \mathrm{~cm}$ from skin, directing downwards in the epidural space (Fig. 2). Then, after a negative test dose of $3 \mathrm{~mL}$ using lignocaine adrenaline mixture (5:1) and a bolus dose $(6 \mathrm{~mL})$ of respective study solution was given, the remaining cath-

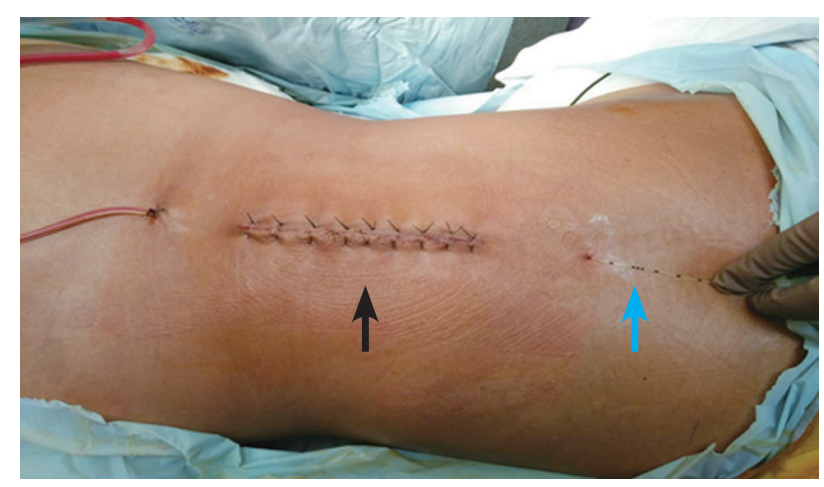

Fig. 2. Subcutaneously tunneled epidural catheter. The catheter at the cranial end with $13 \mathrm{~cm}$ mark at the skin (blue arrow) with $3 \mathrm{~cm}$ inside in the epidural space (black arrow) and $3 \mathrm{~cm}$ away from the incision. Written informed consent for publication of this image was obtained from the patient.

eter was fixed on back using a sterile dressing.

Placement of catheter under direct vision has the distinct advantage of preventing malposition of the catheter and hence maldistribution of the drug.

After shifting the patient in the post-anesthesia care unit (PACU), the following parameters were observed: Total rescue analgesia consumption (injection diclofenac in $\mathrm{mg}$ ), time to first rescue analgesia (in minutes), and patient satisfaction score (range, 1-5) at the end of the ob- 
servation regarding overall treatment.

\section{Statistics}

According to power analysis, at least 30 patients were required in each group to show a difference in postopera- tive analgesic consumption with statistical power $80 \%$ and confidence interval $95 \%$. It was assumed that $70 \%$ of the patients would need rescue analgesic in the group $\mathrm{R}$ during the first 72 hours of the postoperative period. Further, it was estimated that $35 \%$ of the patients will need rescue analgesic in group RD. Therefore, we recruited at least 29

Table 1. Demographic and preoperative details of enrolled patients in both groups

\begin{tabular}{|c|c|c|c|}
\hline Characteristic & Group $R(n=30)$ & Group RD ( $n=30)$ & $p$-value \\
\hline Age (yr) & $41.667 \pm 13.283$ & $39.867 \pm 12.859$ & 0.596 \\
\hline Sex & & & 0.584 \\
\hline Female & 11 & 9 & \\
\hline Male & 19 & 21 & \\
\hline Weight (kg) & $68.167 \pm 10.062$ & $68.733 \pm 8.370$ & 0.813 \\
\hline Height (cm) & $167.2 \pm 6.984$ & $169.5 \pm 6.902$ & 0.205 \\
\hline Body mass index $\left(\mathrm{kg} / \mathrm{m}^{2}\right)$ & $24.443 \pm 3.824$ & $24.068 \pm 3.852$ & 0.707 \\
\hline American Society of Anesthesiologists status & & & 0.398 \\
\hline I & 11 & 8 & \\
\hline$\|$ & 15 & 14 & \\
\hline III & 4 & 8 & \\
\hline \multicolumn{4}{|l|}{ Baseline laboratory parameters } \\
\hline Hemoglobin (g/dL) & $12.69 \pm 1.627$ & $12.22 \pm 1.359$ & 0.230 \\
\hline Platelets (lacs) & $2.653 \pm 0.857$ & $2.556 \pm 0.775$ & 0.650 \\
\hline Total leukocyte count (/mL) & $7,486.66 \pm 1,800$ & $6,883.33 \pm 1,754$ & 0.194 \\
\hline International normalized ratio & $1.147 \pm 0.091$ & $1.149 \pm 0.094$ & 0.912 \\
\hline Prothrombin time index & $88.23 \pm 5.91$ & $88.03 \pm 5.24$ & 0.890 \\
\hline Urea (mg/dL) & $22.33 \pm 6.603$ & $23.23 \pm 5.781$ & 0.577 \\
\hline Creatinine & $0.870 \pm 0.139$ & $0.857 \pm 0.14$ & 0.714 \\
\hline \multicolumn{4}{|l|}{ Preoperative diagnosis } \\
\hline Vertebral fracture & $15(50)$ & $15(50)$ & \\
\hline Cauda equina syndrome & $12(40)$ & $10(33)$ & \\
\hline Canal stenosis & $2(6.6)$ & $3(10)$ & \\
\hline Spondylolisthesis & 0 & $1(3.3)$ & \\
\hline Prolapsed intervertebral disk & $1(3.3)$ & 0 & \\
\hline Compression myelopathy & $1(3.3)$ & 0 & \\
\hline Duration of surgery (min) & $250.00 \pm 50.990$ & $249.833 \pm 37.335$ & 0.989 \\
\hline Duration of anesthesia (min) & $329.66 \pm 70.612$ & $316.33 \pm 41.852$ & 0.377 \\
\hline Baseline systolic blood pressure (mm Hg) & $137.20 \pm 15.949$ & $139.40 \pm 16.684$ & 0.604 \\
\hline Baseline diastolic blood pressure $(\mathrm{mm} \mathrm{Hg})$ & $77.30 \pm 9.154$ & $73.80 \pm 9.323$ & 0.148 \\
\hline Baseline mean arterial pressure $(\mathrm{mm} \mathrm{Hg})$ & $97.26 \pm 6.538$ & $95.66 \pm 6.824$ & 0.358 \\
\hline Baseline heart rate (/min) & $90.26 \pm 6.039$ & $88.16 \pm 5.669$ & 0.170 \\
\hline Baseline peripheral oxygen saturation (\%) & $99.00 \pm 0.742$ & $99.16 \pm 0.833$ & 0.417 \\
\hline Baseline Visual Analog Scale score & $6.73 \pm 0.784$ & $6.43 \pm 0.858$ & 0.163 \\
\hline
\end{tabular}

Values are presented as mean \pm standard deviation or number $(\%)$. R group: epidural ropivacaine only; RD group: epidural ropivacaine with dexmedetomidine. 
patients in each group with $80 \%$ power of study, $5 \%$ significance level, and 5\% superiority margin. Anticipating $5 \%$ of loss to follow-up, we planned to recruit 31 patients in each group. We used following superiority formula for sample size estimation:

$$
N=2 \times\left(\frac{z_{1-\alpha}+z_{1-\beta}}{\delta-\delta_{0}}\right)^{2} \times s^{2}
$$

The obtained data were entered in an MS excel spreadsheet (Microsoft Corp., Redmond, WA, USA). Data were analyzed using IBM SPSS ver. 21.0 (IBM Corp., Armonk, NY, USA). Categorical data are represented as frequencies and percentages. Continuous data are shown as mean and standard deviation values. Unpaired $t$-test (parametric test) was used as test of significance for continuous, normal data. Mann-Whitney test (non-parametric test) was used as test of significance for continuous, non-normal data. Chi-square test was used for testing the significance of difference in categorical data. Bar diagrams and line diagrams were made wherever needed. Time trend graph was utilized to visualize the rate of change in the VAS score. Inferential analysis was performed for numerical data using repeated measures analysis of variance or other appropriate technique. The $p$-value was determined to finally evaluate the levels of significance. A $p$-value $<0.05$ was considered to indicate a significance of $5 \%$.

\section{Results}

There was no difference in the demographic characteristics of the two groups ( $p$-value $>0.05$ ). The enrolled patients were found comparable, with respect to the preoperative laboratory parameters, surgical diagnosis, preoperative mean VAS score, and mean surgical duration (Table 1). There were 19, 29, and 12 ASA I, ASA II, and ASA III patients, respectively, in our study. In addition, there was no significant difference in the distribution of the ASA grade between the two groups ( $p>0.05)$. The mean \pm standard deviation value of total rescue analgesia consumption was $162.5 \pm 68.4$ in the RD group and $247.5 \pm 48.8 \mathrm{mg}$ in the $\mathrm{R}$ group (Table 2). The mean value of rescue analgesia consumption was more in the $\mathrm{R}$ group than in the RD group at postoperative day (POD) 0,1 , and 2 as well as total consumption. This difference was significant in unpaired $t$-test $(p<0.05)$. Mean time to first rescue analgesia in the $\mathrm{RD}$ group was $594.6 \pm 83.0$ minutes and that in the R group was 103.6 \pm 53.2 minutes (Table 2). Therefore, the addition of dexmedetomidine increased the pain-free duration in the $\mathrm{RD}$ group. The unpaired $t$-test showed a significant $(p<0.05)$. The mean patient satisfaction score was $4.2 \pm 0.7$ in the RD group and $3.2 \pm 0.6$ in the R group (Table 2). Thus, the mean patient satisfactory score at 72 hours was more in the $\mathrm{RD}$ group than in the $\mathrm{R}$ group. Unpaired $t$-test showed that this difference was significant $(p<0.05)$.

As shown in Fig. 3, the mean VAS score of R group was higher than that of the RD group from 0-72 hours. In most readings, the difference between the two groups was significant on unpaired $t$-test $(p<0.05)$.

Hemodynamic parameters (mean arterial pressure [MAP], heart rate [HR], and others) were measured in the postoperative period (from 0-72 hours postoperatively). A comparison of the differences in the mean values of MAP and HR between the two groups at different time

Table 2. Postoperative observed parameters of enrolled patients in both groups

\begin{tabular}{lccc} 
Variable & Group R $(\mathrm{n}=30)$ & Group RD (n=30) & $p$-value \\
\hline Total rescue analgesia consumption in POD $0(\mathrm{mg})$ & $110.00 \pm 38.05$ & $75.00 \pm 00.00$ & 0.596 \\
\hline Total rescue analgesia consumption in POD $1(\mathrm{mg})$ & $72.50 \pm 46.12$ & $47.50 \pm 36.75$ & $0.024^{*}$ \\
\hline Total rescue analgesia consumption in POD $2(\mathrm{mg})$ & $65.00 \pm 42.85$ & $40.00 \pm 38.05$ & $0.020^{*}$ \\
\hline Total rescue analgesia consumption in 72 hr $(\mathrm{mg})$ & $247.50 \pm 48.84$ & $162.50 \pm 68.46$ & $0.001^{*}$ \\
\hline Mean time to first rescue analgesia $(\mathrm{min})$ & $103.66 \pm 53.28$ & $594.66 \pm 83.03$ & $0.001^{*}$ \\
\hline Mean value of patient satisfaction scores & $3.233 \pm 0.678$ & $4.233 \pm 0.727$ & $0.001^{*}$ \\
\hline Mean value of post-anesthesia care unit stays $(\mathrm{min})$ & $316.16 \pm 41.11$ & $252.83 \pm 38.00$ & $0.001^{*}$ \\
\hline Mean value of hospital stays (min) & $5.128 \pm 417.10$ & $5,164 \pm 412.07$ & 0.738 \\
\hline
\end{tabular}

Values are presented as mean \pm standard deviation or number (\%). R group: epidural ropivacaine only; RD group: epidural ropivacaine with dexmedetomidine. POD, postoperative day.

${ }^{*} p<0.05$. 


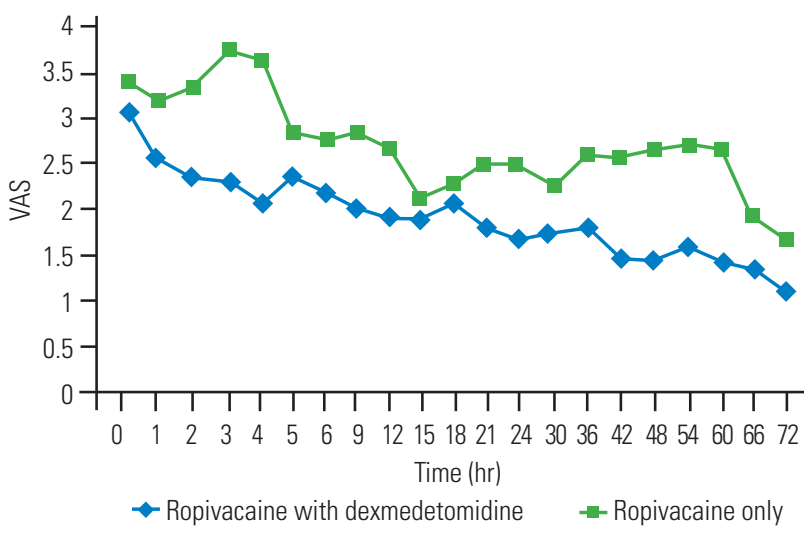

Fig. 3. Mean VAS at different time interval. VAS, Visual Analog Scale.
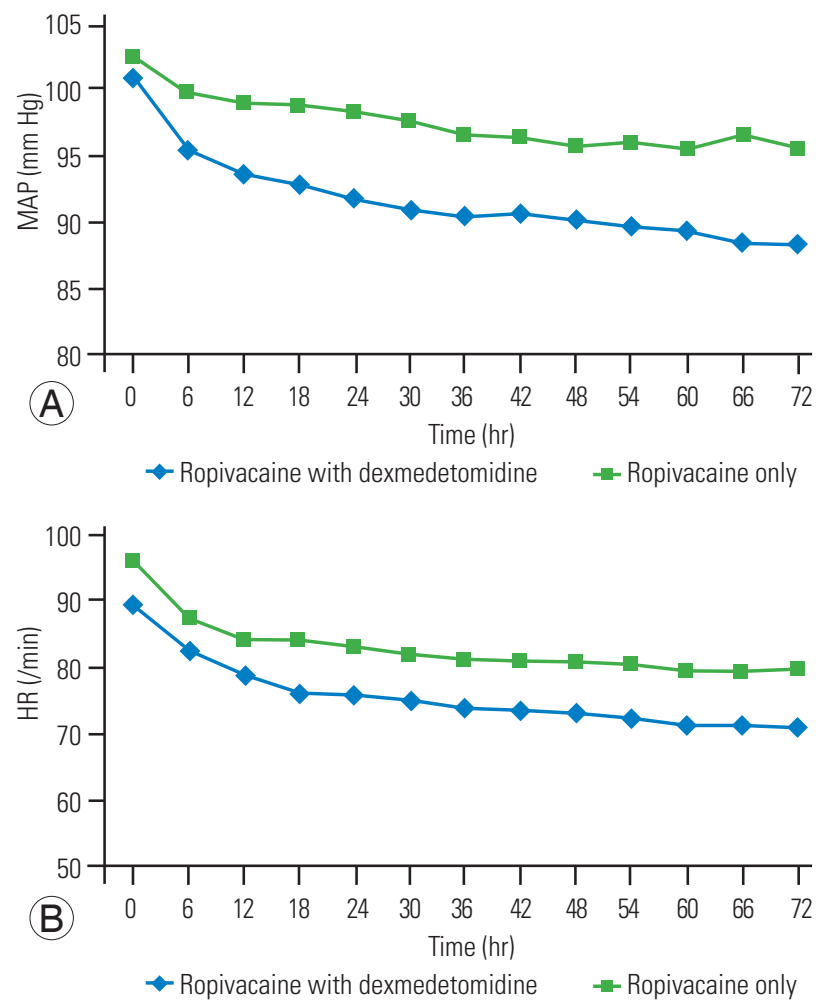

Fig. 4. (A) Mean MAP (mm Hg) at different time interval. (B) Mean HR (/min) at different time intervals. MAP, mean arterial pressure; $H R$, heart rate.

intervals showed significant difference on unpaired $t$-test at most observational points $(p<0.05)$ (Fig. 4A, B).

As shown in Fig. 5, the mean sedation score was more in the $\mathrm{RD}$ group than in the $\mathrm{R}$ group from $0-48$ hours. At all the points of recording, the difference between the two groups was significant on unpaired $t$-test $(p<0.05)$. At POD $0,36.7 \%(n=11)$ in the R group and $20 \%(n=6)$ in the RD group had nausea. However, this difference was not significant on chi-square test $(p>0.05)$. At POD $0,10 \%$

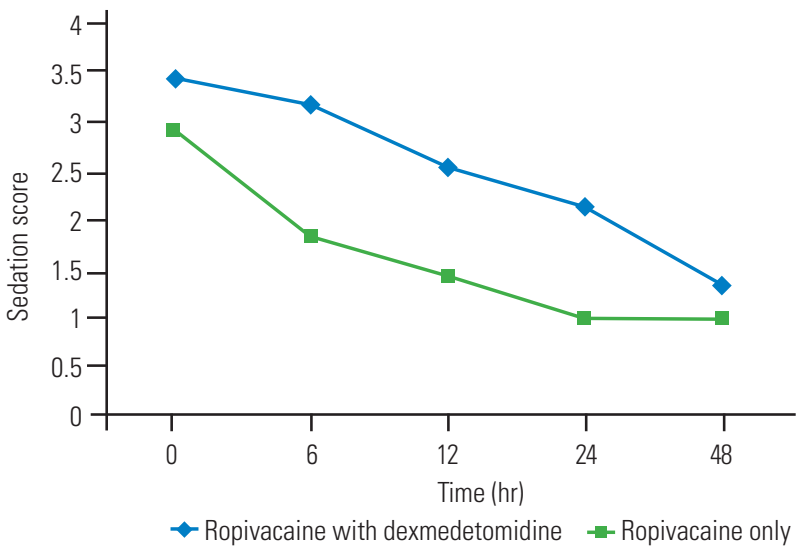

Fig. 5. Mean sedation scores.

$(\mathrm{n}=3)$ in the R group and $6.7 \%(\mathrm{n}=2)$ in the $\mathrm{RD}$ group had vomiting. At POD 1, 13.3\% ( $\mathrm{n}=4)$ in the R group and $10 \%(\mathrm{n}=3)$ in the $\mathrm{RD}$ group had vomiting. However, this difference was not significant on chi-square test $(p>0.05)$. We found a significant lower duration of PACU stay in the $\mathrm{RD}$ group than in the $\mathrm{R}$ group $(252.8 \pm 38.0$ minutes versus $316.1 \pm 41.1$ minutes) with an estimated mean difference of $-63.3(-83.7$ to -42.8$)$.

\section{Discussion}

Computerization, industrialization, motorization, and aging population are the four key factors responsible for the increase in spine injuries in the previous few decades. Increase in motor vehicles, poor road designs, and lower compliance of young population to traffic laws, as evident by increased traffic challans and hospitalizations are important factors that have increased road traffic accidents and subsequently spine injuries in India [8]. State-of-theart training centers with advance diagnostic system and fellowship/training courses are responsible for increase in spine surgeries in India [9]. It is important to treat postoperative pain because it leads to impaired activity and function, thus increasing the healthcare cost $[3,10]$. In addition, the treatment of postoperative pain is associated with better results in terms of early mobilization, early rehabilitation, and complication prevention, such as deep vein thrombosis, pulmonary embolism, pneumonia, and atelectasis [11].

We used injection diclofenac sodium $75 \mathrm{mg}$ as the rescue analgesic in the postoperative period; $6 \mathrm{~mL}$ of respective epidural study solution bolus was given if pain was not relieved by diclofenac within 20 minutes. 
Our study revealed that the patients in $\mathrm{RD}$ group (ropivacaine with dexmedetomidine) had decreased rescue analgesia consumption at POD 0, 1,2 and subsequently total rescue analgesia consumption than those in the $\mathrm{R}$ group (ropivacaine only). We found that the mean total rescue analgesia consumption (injection diclofenac in $\mathrm{mg}$ ) was significantly lower in the $\mathrm{RD}$ group than in the $\mathrm{R}$ group (162.5 $\pm 68.4 \mathrm{mg}$ versus $247 \pm 48.8 \mathrm{mg}, p<0.05)$. The mean time to first rescue analgesia in minutes was significantly delayed in the $\mathrm{RD}$ group as compared to that in the $\mathrm{R}$ group (594.6 \pm 83.0 minutes versus $103.6 \pm 53.2$ minutes). This finding is essentially explained by the effect of dexmedetomidine at the spinal, supra-spinal, and peripheral nerves leading to analgesia by suppression of $\mathrm{C}$ fibers and hyperpolarization of posterior horn neurons [12].

Our findings correlated with previously published studies using epidural dexmedetomidine. Saravana Babu et al. [13] compared epidural ropivacaine with dexmedetomidine and ropivacaine with clonidine in patients who were undergoing spine surgeries. They showed that patients who received epidural ropivacaine with dexmedetomidine had an earlier onset and prolonged duration of analgesia. They gave a $20-\mathrm{mL}$ epidural bolus of the respective study solution whenever the patient complained of postoperative pain (VAS score $>4$ ), and their study ended whenever the patient demanded a second analgesic [13]. In contrast, we provided continuous infusion of epidural drug (s) over 48 hours to avoid any hemodynamic compromise associated with bolus dosing.

Hetta et al. [14] conducted a study that compared continuous infusions ( 48 hours) of epidural bupivacaine $0.1 \%$ versus bupivacaine $0.1 \%$ with dexmedetomidine $0.5 \mu \mathrm{g} /$ $\mathrm{mL}$ in patients undergoing abdominal cancer surgery and concluded that the addition of dexmedetomidine in epidural route significantly reduced the morphine consumption, delayed time to first rescue analgesia, and reduced pain scores during postoperative 48 hours [14]. In another study on 100 patients undergoing lower limb surgery, dexmedetomidine was compared with fentanyl as an epidural adjuvant and showed better hemodynamic stability, prolonged postoperative analgesia, and better sedation levels [15]. A recent meta-analysis of 12 randomized controlled trial was performed by Zhang et al. [16]. In their analysis, compared to the control group, the epidural dexmedetomidine group had reduced rescue analgesia consumption, increased duration of analgesia, and better sedation scores with insignificant differences in hemodynamic events, such as bradycardia and hypotension [16].

Chiruvella et al. [17] also observed that dexmedetomidine $1 \mu \mathrm{g} / \mathrm{kg}$ was a better neuraxial adjuvant to levobupivacaine $0.125 \%$ when compared to clonidine $2 \mu \mathrm{g} / \mathrm{kg}$ for providing early onset and prolonged postoperative epidural analgesia with stable cardiorespiratory parameters in total abdominal hysterectomies. Soni [18] showed that the addition of dexmedetomidine to ropivacaine in epidural anesthesia achieved faster onset and longer duration of sensory and motor blockade. Bajwa et al. [19] also observed similar results and concluded that epidural dexmedetomidine is a better adjuvant than clonidine in terms of patient comfort, stable cardio-respiratory parameters, as well as intraoperative and postoperative analgesia as compared to epidural clonidine in the vaginal hysterectomy surgeries. Salgado et al. [20] also concluded that epidural dexmedetomidine did not affect the onset time or upper level of anesthesia; however, it prolonged the sensory and motor block duration time and postoperative analgesia. They also suggested the clear synergism between epidural dexmedetomidine and ropivacaine and that this drug association does not bring about additional morbidity [20].

We also measured the patient satisfaction scores based on the Likert scale; a score of 1 implied lowest satisfaction and 5 implied highest satisfaction with regard to overall treatment. Similar to previous studies with epidural analgesia in spine surgeries, our study also showed satisfaction in both groups $[3,21]$. However, the satisfaction was significantly higher in the $\mathrm{RD}$ group $(4.2 \pm 0.7$ versus $3.2 \pm 0.6)$.

As suggested previously in human studies, the decrease in HR and MAP with dexmedetomidine could be due to central as well as peripheral sympatholysis or cardiac vagal activity [13]. We kept concentration of dexmedetomidine in infusion to an optimum level of $1 \mu \mathrm{g} / \mathrm{mL}$ and did not use bolus dosing except for the initial $6 \mathrm{~mL}$ of study solution that was given after a negative test dose.

No patient in our study developed additional motor blockade that could be attributed to epidural administration of local anesthetic ropivacaine because we did not use a concentration of $>0.1 \%$ ropivacaine in any case. As per previous studies, $0.1 \%$ concentration of ropivacaine did not cause additional motor blockade (Table 3) [22].

We found lower incidences of postoperative nausea and vomiting (PONV) in the $\mathrm{RD}$ group then in the $\mathrm{R}$ group at POD 0 and 1; there was no incidence of PONV on POD 2 ; however, these finding were not significant $(p>0.05)$. Our incidence of PONV agreed with an earlier published 
Table 3. Details of perioperative motor power of LLs during study

\begin{tabular}{|c|c|c|c|c|c|c|c|}
\hline \multirow{2}{*}{ Variable } & \multirow{2}{*}{ Preoperative } & \multicolumn{6}{|c|}{ Time (hr) } \\
\hline & & 3 & 6 & 12 & 24 & 48 & 72 \\
\hline \multicolumn{8}{|l|}{ RD group } \\
\hline \multicolumn{8}{|c|}{ Motor power in Rt LL } \\
\hline M2 & 2 & 2 & 2 & 2 & 1 & 1 & 0 \\
\hline M3 & 5 & 5 & 5 & 5 & 6 & 6 & 5 \\
\hline M4 & 8 & 8 & 8 & 8 & 8 & 8 & 10 \\
\hline M5 & 15 & 15 & 15 & 15 & 15 & 15 & 15 \\
\hline \multicolumn{8}{|c|}{ Motor power in Lt LL } \\
\hline M2 & 1 & 1 & 1 & 1 & 1 & 1 & 0 \\
\hline M3 & 7 & 7 & 7 & 7 & 7 & 7 & 8 \\
\hline M4 & 11 & 11 & 11 & 11 & 11 & 11 & 11 \\
\hline M5 & 11 & 11 & 11 & 11 & 11 & 11 & 11 \\
\hline \multicolumn{8}{|l|}{ R group } \\
\hline \multicolumn{8}{|c|}{ Motor power of Rt LL } \\
\hline M2 & 0 & 0 & 0 & 0 & 0 & 0 & 0 \\
\hline M3 & 6 & 6 & 6 & 6 & 5 & 5 & 3 \\
\hline M4 & 11 & 11 & 11 & 11 & 12 & 12 & 14 \\
\hline M5 & 13 & 13 & 13 & 13 & 13 & 13 & 13 \\
\hline \multicolumn{8}{|c|}{ Motor power of Lt LL } \\
\hline M2 & 0 & 0 & 0 & 0 & 0 & 0 & 0 \\
\hline M3 & 6 & 6 & 6 & 6 & 6 & 5 & 4 \\
\hline M4 & 8 & 8 & 8 & 8 & 8 & 9 & 8 \\
\hline M5 & 16 & 16 & 16 & 16 & 16 & 16 & 18 \\
\hline
\end{tabular}

Values are presented as number of patients. $R$ group: epidural ropivacaine only; $\mathrm{RD}$ group: epidural ropivacaine with dexmedetomidine.

Rt, right; Lt, left; LL, lower limb.

meta-analysis that demonstrated that intravenous dexmedetomidine significantly reduced the incidence of PONV [23]. The results obtained in our study may represent systemic absorption of dexmedetomidine given via an epidural infusion over 48 hours.

Pain and PONV are the two key predictors of length of PACU stays after surgery [24]. We found a significant lower duration of PACU stay in the RD group than in the R group (252.8 \pm 38.0 minutes versus $316.1 \pm 41.1 \mathrm{~min}$ utes) with an estimated mean difference of $-63.3(-83.7$ to $-42.8)$.

None of the enrolled patient in our study had any epidural catheter-related complications, such as misplacement, dislodgement, blockage, breakage, and catheter-site infections.

\section{Conclusions}

We conclude that in patients undergoing thoracolumbar spine instrumentation, epidural ropivacaine with dexmedetomidine is associated with a significantly lower amount of rescue analgesia consumption and higher patient satisfaction scores than epidural ropivacaine only. However, further prospective trials that consider preoperative pain and analgesics consumption are needed. Epidural dexmedetomidine can serve as an excellent adjuvant for continuous infusion in the postoperative period.

\section{Conflict of Interest}

No potential conflict of interest relevant to this article was reported.

\section{Author Contributions}

Faisal Quershi: conduct the whole study and writing the manuscript; Shyam Charan Meena: design, conduct the study, and writing the final manuscript; Vishal Kumar: conduct the study and help in statistical analysis; and Kajal Jain: analysis the observation and help in writing the manuscript.

\section{References}

1. Rajaee SS, Bae HW, Kanim LE, Delamarter RB. Spinal fusion in the United States: analysis of trends from 1998 to 2008. Spine (Phila Pa 1976) 2012;37:6776.

2. Bianconi M, Ferraro L, Ricci R, et al. The pharmacokinetics and efficacy of ropivacaine continuous wound instillation after spine fusion surgery. Anesth Analg 2004;98:166-72.

3. Gottschalk A, Freitag M, Tank S, et al. Quality of postoperative pain using an intraoperatively placed epidural catheter after major lumbar spinal surgery. Anesthesiology 2004;101:175-80.

4. Sandkuhler J. Fear the pain. Lancet 2002;360:426.

5. Benyamin R, Trescot AM, Datta S, et al. Opioid complications and side effects. Pain Physician 2008;11:S105-20.

6. Polley LS, Columb MO, Naughton NN, Wagner DS, van de Ven CJ. Relative analgesic potencies of ropivacaine and bupivacaine for epidural analgesia in labor: 
implications for therapeutic indexes. Anesthesiology 1999;90:944-50.

7. Dyck JB, Maze M, Haack C, Vuorilehto L, Shafer SL. The pharmacokinetics and hemodynamic effects of intravenous and intramuscular dexmedetomidine hydrochloride in adult human volunteers. Anesthesiology 1993;78:813-20.

8. Gururaj G. Road traffic deaths, injuries and disabilities in India: current scenario. Natl Med J India 2008;21:14-20.

9. Mulukutla RD. From plaster beds to robotics... evolution of spine surgery in India. Indian Spine J 2019;2:111.

10. Kehlet $\mathrm{H}$, Holte K. Effect of postoperative analgesia on surgical outcome. Br J Anaesth 2001;87:62-72.

11. Gan TJ. Poorly controlled postoperative pain: prevalence, consequences, and prevention. J Pain Res 2017;10:2287-98.

12. Guo TZ, Jiang JY, Buttermann AE, Maze M. Dexmedetomidine injection into the locus ceruleus produces antinociception. Anesthesiology 1996;84:873-81.

13. Saravana Babu M, Verma AK, Agarwal A, Tyagi CM, Upadhyay M, Tripathi S. A comparative study in the post-operative spine surgeries: epidural ropivacaine with dexmedetomidine and ropivacaine with clonidine for post-operative analgesia. Indian J Anaesth 2013;57:371-6.

14. Hetta DF, Fares KM, Abedalmohsen AM, AbdelWahab AH, Elfadl GM, Ali WN. Epidural dexmedetomidine infusion for perioperative analgesia in patients undergoing abdominal cancer surgery: randomized trial. J Pain Res 2018;11:2675-85.

15. Bajwa SJ, Arora V, Kaur J, Singh A, Parmar SS. Comparative evaluation of dexmedetomidine and fentanyl for epidural analgesia in lower limb orthopedic surgeries. Saudi J Anaesth 2011;5:365-70.

16. Zhang X, Wang D, Shi M, Luo Y. Efficacy and safety of dexmedetomidine as an adjuvant in epidural anal- gesia and anesthesia: a systematic review and metaanalysis of randomized controlled trials. Clin Drug Investig 2017;37:343-54.

17. Chiruvella S, Donthu B, Nallam SR, Salla DB. Postoperative analgesia with epidural dexmedetomidine compared with clonidine following total abdominal hysterectomies: a prospective double-blind randomized trial. Anesth Essays Res 2018;12:103-8.

18. Soni P. Comparative study for better adjuvant with ropivacaine in epidural anesthesia. Anesth Essays Res 2016;10:218-22.

19. Bajwa SJ, Bajwa SK, Kaur J, et al. Dexmedetomidine and clonidine in epidural anaesthesia: a comparative evaluation. Indian J Anaesth 2011;55:116-21.

20. Salgado PF, Sabbag AT, Silva PC, et al. Synergistic effect between dexmedetomidine and $0.75 \%$ ropivacaine in epidural anesthesia. Rev Assoc Med Bras (1992) 2008;54:110-5.

21. Fisher CG, Belanger L, Gofton EG, et al. Prospective randomized clinical trial comparing patient-controlled intravenous analgesia with patient-controlled epidural analgesia after lumbar spinal fusion. Spine (Phila Pa 1976) 2003;28:739-43.

22. Scott DA, Chamley DM, Mooney PH, Deam RK, Mark AH, Hagglof B. Epidural ropivacaine infusion for postoperative analgesia after major lower abdominal surgery: a dose finding study. Anesth Analg 1995;81:982-6.

23. Jin S, Liang DD, Chen C, Zhang M, Wang J. Dexmedetomidine prevent postoperative nausea and vomiting on patients during general anesthesia: a PRISMAcompliant meta analysis of randomized controlled trials. Medicine (Baltimore) 2017;96:e5770.

24. Ganter MT, Blumenthal S, Dubendorfer S, et al. The length of stay in the post-anaesthesia care unit correlates with pain intensity, nausea and vomiting on arrival. Perioper Med (Lond) 2014;3:10. 